\title{
Partial Differential Equations that are Hard to Classify
}

\author{
HOWISON S. D. ${ }^{1}$, LACEY A. A. ${ }^{2, *}$ and OCKENDON J. R. ${ }^{1}$ \\ 1 OCIAM, Mathematical Institute, University of Oxford, 24-29 St. Giles', Oxford, \\ OX1 3LB, UK. \\ ${ }^{2}$ Maxwell Institute for Mathematical Sciences, and School of Mathematical and \\ Computer Sciences, Heriot-Watt University, Riccarton, Edinburgh, EH14 4AS, UK.
}

Received 10 June 2011; Accepted 24 November 2011

\begin{abstract}
Semi-linear $n \times n$ systems of the form $\mathbf{A} \partial \mathbf{u} / \partial x+\mathbf{B} \partial \mathbf{u} / \partial y=\mathbf{f}$ can generally be solved, at least locally, provided data are imposed on non-characteristic curves. There are at most $n$ characteristic curves and they are determined by the coefficient matrices on the left-hand sides of the equations. We consider cases where such problems become degenerate as a result of ambiguity associated with the definition of characteristic curves. In such cases, the existence of solutions requires restrictions on the data and solutions might not be unique.
\end{abstract}

AMS Subject Classifications: 35A21, 35A30, 35E20, 35N05

Chinese Library Classifications: 0175.2

Key Words: Linear systems of first-order PDEs; classification; canonical systems.

\section{Introduction}

It is well known that the Cauchy-Kowalevski Theorem tells us that a problem of the form

$$
\mathbf{A} \frac{\partial \mathbf{u}}{\partial x}+\mathbf{B} \frac{\partial \mathbf{u}}{\partial y}=\mathbf{f}
$$

where $\mathbf{u}$ is an $n$-dimensional vector and $\mathbf{A}$ and $\mathbf{B}$ are $n \times n$ constant matrices, has an analytic solution, at least locally, provided we have analytic data on a non-characteristic analytic curve. The unique solution can be determined, locally, by solving $n$ scalar equations given by (1.1), in conjunction with the $n$ found by differentiating the Cauchy data

$$
\mathbf{u}=\mathbf{U}_{0}(t) \quad \text { on } \mathbf{x}=\mathbf{x}_{0}(t)
$$
*Corresponding author. Email addresses: howison@maths.ox.ac.uk (S. D. Howison), A. A. Lacey@hw.ac.uk
(A. A. Lacey), ock@maths.ox.ac.uk (J. R. Ockendon) 
along the curve $(x, y)=\mathbf{x}=\mathbf{x}_{0}(t)=\left(x_{0}(t), y_{0}(t)\right)$, to find the $2 n$ first partial derivatives $\partial \mathbf{u} / \partial x$ and $\partial \mathbf{u} / \partial y$. An entirely equivalent way of thinking about characteristics is to regard them as curves across which $\mathbf{u}$ can have discontinuous first derivatives.

The Cauchy-Kowalevski argument fails when the curve is characteristic so that

$$
\lambda=\frac{\mathrm{d} x}{\mathrm{~d} t}, \quad \mu=\frac{\mathrm{d} y}{\mathrm{~d} t} \quad \text { (not both zero) }
$$

are such that (1.1) together with the equations got from differentiating (1.2), in vector form

$$
\lambda \frac{\partial \mathbf{u}}{\partial x}+\mu \frac{\partial \mathbf{u}}{\partial y}=\mathbf{U}_{0}^{\prime}
$$

fail to have a unique solution. This of course happens with $\lambda, \mu$ such that

$$
\left|\begin{array}{c|c}
\mathbf{A} & \mathbf{B} \\
\hline \lambda \mathbf{I} & \mu \mathbf{I}
\end{array}\right|=\left|\begin{array}{cccccc}
a_{11} & \ldots & a_{1 n} & b_{11} & \ldots & b_{1 n} \\
\vdots & & \vdots & \vdots & & \vdots \\
a_{n 1} & \ldots & a_{n n} & b_{n 1} & \ldots & b_{n n} \\
\lambda & \ldots & 0 & \mu & \ldots & 0 \\
\vdots & \ddots & \vdots & \vdots & \ddots & \vdots \\
0 & \ldots & \lambda & 0 & \ldots & \mu
\end{array}\right|=0,
$$

where $\mathbf{I}$ is the $n \times n$ identity matrix. Equivalently,

$$
\left|\begin{array}{cccccc}
\mu a_{11}-\lambda b_{11} & \ldots & \mu a_{1 n}-\lambda b_{1 n} & b_{11} & \ldots & b_{1 n} \\
\vdots & & \vdots & \vdots & & \vdots \\
\mu a_{n 1}-\lambda b_{n 1} & \ldots & \mu a_{n n}-\lambda b_{n n} & b_{n 1} & \ldots & b_{n n} \\
0 & \ldots & 0 & 1 & \ldots & 0 \\
\vdots & \ddots & \vdots & \vdots & \ddots & \vdots \\
0 & \ldots & 0 & 0 & \ldots & 1
\end{array}\right|=|\mu \mathbf{A}-\lambda \mathbf{B}|=0
$$

For "most" problems, with no sort of degeneracy associated with the left-hand side of (1.1), the condition (1.5) would make the curve direction $(\lambda, \mu)$ that of the characteristic.

In the present paper we consider problems such that (1.5) holds for all $\lambda, \mu$, so that, whatever direction is used, the system (1.1) fails to have a unique solution. We anticipate that, since the coefficient matrix of the combined system

$$
\left(\begin{array}{c|c}
\mathbf{A} & \mathbf{B} \\
\hline \lambda \mathbf{I} & \mu \mathbf{I}
\end{array}\right)\left(\frac{\partial \mathbf{u} / \partial x}{\partial \mathbf{u} / \partial y}\right)=\left(\begin{array}{c}
\mathbf{f} \\
\mathbf{U}_{0}^{\prime}
\end{array}\right)
$$

is singular, whatever data curve is chosen, at least one compatibility condition relating $\mathbf{f}$ and $\mathbf{u}_{0}$ has to be satisfied if the problem (1.1), (1.2) is to have a solution; moreover, that if this condition holds, the problem can have multiple solutions. It is clear that degeneracy is associated with the rank of $\mu \mathbf{A}-\lambda \mathbf{B}$ being identically less than $n$. 
We remark that the above comments and conditions apply both when we seek curves across which $\partial \mathbf{u} / \partial x$ and $\partial \mathbf{u} / \partial y$ have jump discontinuities while $\mathbf{u}$ remains continuous, and when we consider weak solutions for systems in which $\mathbf{u}$ itself has a jump discontinuity. Indeed, this observation is one of the motivations for the present paper.

Although situations in which (1.6) holds for all $\lambda$ and $\mu$ are not usually discussed in partial differential equations texts, either from the analytical or numerical viewpoints, they can readily occur in practice. For example, consider a simple normalised model for longitudinal elastic waves where $t$ is the time, $x$ the displacement, $u$ the velocity, $\sigma$ the stress and $X$ the Lagrangian coordinate relative to the unstressed state:

$$
\left(\begin{array}{lll}
1 & 0 & 0 \\
0 & 1 & 0 \\
0 & 0 & 0
\end{array}\right) \frac{\partial}{\partial t}\left(\begin{array}{l}
x \\
u \\
\sigma
\end{array}\right)+\left(\begin{array}{ccc}
0 & 0 & 0 \\
0 & 0 & -1 \\
1 & 0 & 0
\end{array}\right) \frac{\partial}{\partial X}\left(\begin{array}{l}
x \\
u \\
\sigma
\end{array}\right)=\left(\begin{array}{l}
u \\
0 \\
\sigma
\end{array}\right) .
$$

In this case the matrix analogous to that in (1.6) has rank 2. We note that while trivial manipulations reveal that any one of the dependent variables satisfies the scalar wave equation with wave velocities \pm 1 , (1.6) tells us nothing about the wave speeds. Of even more concern is the fact that if the last scalar equation is generalised to the visco-elastic law $\partial x / \partial X-\sigma=\epsilon \partial \sigma / \partial t$, then (1.6) gives $\epsilon \lambda^{3}=0$, suggesting that jump discontinuities in the derivatives only occur on $t=$ constant lines for $\epsilon \rightarrow 0$.

Another common situation concerns the derivation of Charpit's equations for scalar non-quasi-linear first-order equations of the form

$$
F\left(x, y, u, \frac{\partial u}{\partial x}, \frac{\partial u}{\partial y}\right)=0
$$

As in [1], for example, five different quasi-linear equations can easily be written down for the vector $(u, p, q)$, where $p=\partial u / \partial x, q=\partial u / \partial y$, and most subsets of three of these five equations satisfy (1.6) identically.

An easily understood example with three independent variables is $\operatorname{curl}(u, v, w)^{\top}=\mathbf{0}$. Then

$$
\left(\mathbf{A}_{1} \frac{\partial}{\partial x}+\mathbf{A}_{2} \frac{\partial}{\partial y}+\mathbf{A}_{3} \frac{\partial}{\partial z}\right)\left(\begin{array}{c}
u \\
v \\
w
\end{array}\right)=\mathbf{0}
$$

where the generalisation of (1.6), namely

$$
\sum_{1}^{3} \xi_{i} \mathbf{A}_{i}=\left(\begin{array}{ccc}
0 & -\xi_{3} & \xi_{2} \\
\xi_{3} & 0 & -\xi_{1} \\
-\xi_{2} & \xi_{1} & 0
\end{array}\right)
$$

has rank 2. (This result gives no hint that $(u, v, w)^{\top}$ is a gradient.)

Denoting the number of scalar equations by $n_{1}$, the number of scalar dependent variables by $n_{2}$ and the number of independent variables by $n_{3}$, other $n_{1} \times n_{2} \times n_{3}$ examples are: 
(i) Elastic waves in plane strain and in three dimensions.

Here there are three stress components, two displacements, all functions of $(x, y, t)$ and we have a $7 \times 7 \times 3$ system in which, in the notation of (1.10), the $7 \times 7$ matrix $\sum_{1}^{3} \xi_{i} \mathbf{A}_{i}$ has rank 4 .

The analogous $12 \times 12$ matrix for general three-dimensional waves described by a $12 \times 12 \times 4$ system has rank 6 .

(ii) Maxwell's equations.

Considering the $8 \times 6 \times 4$ system

$$
\operatorname{curl} \mathbf{H}=\epsilon \frac{\partial \mathbf{E}}{\partial t}, \quad \operatorname{curl} \mathbf{E}=-\mu \frac{\partial \mathbf{H}}{\partial t}, \quad \operatorname{div} \mathbf{E}=0, \quad \operatorname{div} \mathbf{H}=0,
$$

we find that the four $8 \times 6$ matrices $\mathbf{A}_{i}$ are such that $\sum_{1}^{4} \xi \mathbf{A}_{i}$ has rank 6 . However, choosing just six equations to get a $6 \times 6 \times 4$ system with four $6 \times 6$ matrices $\mathbf{A}_{i}$, the rank can be either 5 or 6 , depending on which two equations are dropped; with a "wrong" selection the system is degenerate. The full $8 \times 6 \times 4$ is non-degenerate, but over-determined. (See [2] for a discussion of over-determined systems.)

(iii) The reduction of general elliptic systems to first-order systems.

When the elliptic equation $\frac{\partial^{2} u}{\partial x^{2}}+\frac{\partial^{2} u}{\partial y^{2}}=0$ is replaced by

$$
\frac{\partial u_{1}}{\partial x}+\frac{\partial u_{2}}{\partial y}=0, \quad \frac{\partial u}{\partial x}-u_{1}=0, \quad \frac{\partial u}{\partial y}-u_{2}=0,
$$

we obtain a degenerate system for $\mathbf{u}=\left(u, u_{1}, u_{2}\right)^{\top}$ in which

$$
\mathbf{A}=\left(\begin{array}{lll}
0 & 1 & 0 \\
1 & 0 & 0 \\
0 & 0 & 0
\end{array}\right), \quad \mathbf{B}=\left(\begin{array}{lll}
0 & 0 & 1 \\
0 & 0 & 0 \\
1 & 0 & 0
\end{array}\right)
$$

Such systems have been treated in [3], [4] by allocating "weights" to both differentiated and undifferentiated terms, and this always results in determinantal criteria that do not degenerate. In the above example, the weights for the undifferentiated terms $-u_{1}$ and $-u_{2}$ are equal to the coefficients of $u_{1}$ and $u_{2}$, in this case both equal to -1 and the matrix $\mu \mathbf{A}-\lambda \mathbf{B}$ is replaced by

$$
\left(\begin{array}{ccc}
0 & \mu & -\lambda \\
\mu & -1 & 0 \\
-\lambda & 0 & -1
\end{array}\right)
$$

whose determinant does not vanish for real $\lambda, \mu$. Indeed, in [4] it is proved that, if the original higher-order system is elliptic, then, when suitable weights are introduced, the resulting generalised determinant never vanishes for real parameters $\lambda$, $\mu$. We shall return to this result on pages 50 and 63 . 
For linear systems with constant coefficients, the method of weights is equivalent to that of seeking explicit exponential solutions, which is the approach we will adopt for most of this paper. This will enable us to see, by elementary means, not only how to classify given degenerate first-order systems, but also to identify the types of singularities they can support and appropriate boundary conditions.

We also note that partial-differential-algebraic systems are inevitably degenerate when thought of as quasi-linear systems of partial differential equations and the classification of such systems has been discussed in [5]. While it is tempting to conjecture that degenerate systems are, generally, differential-algebraic, we shall soon find that this is not the case.

\section{General approach}

In the following sections we shall only consider linear and semi-linear problems, with, for simplicity, the coefficient matrices A and B being constant, although some of the results could be generalised to allow them to vary with $x$ and $y$. We take two points of view:

- We first ask whether or not the solutions can have discontinuous first derivatives across any real curves. For simplicity here, we only consider constant-coefficient homogeneous equations of the form

$$
\mathbf{A} \frac{\partial \mathbf{u}}{\partial x}+\mathbf{B} \frac{\partial \mathbf{u}}{\partial y}=\mathbf{C u},
$$

where the matrix $\mathbf{C}$ is also constant. We then consider the result of seeking a solution with gradient discontinuities, of the form

$$
\mathbf{u}(x, y)=\mathrm{e}^{\alpha y-\beta x}\left(\mathcal{H}_{1}(\lambda y-\mu x) \mathbf{u}_{0}+\mathcal{H}_{2}(\lambda y-\mu x) \mathbf{u}_{1}+\cdots\right),
$$

where $\mathcal{H}_{1}(\cdot)$ is the integral if the Heaviside function $\mathcal{H}(\cdot), \mathcal{H}_{2}^{\prime}=\mathcal{H}_{1}$ and so on, and where $\alpha, \beta$ and $\mathbf{u}_{0}, \mathbf{u}_{1}$ etc. are constant. However, as we shall see, $\alpha$ and $\beta$ may often be ignored in a local analysis. More precisely, it is only in cases where we need to consider several terms in (1.13) simultaneously that the exponential dependence needs to be taken into account for the purposes of studying singularity propagation. We shall often refer to $\mathbf{u}_{0}$ as an eigenvector. Note that, as we are only considering two dimensions, we are free to take $\alpha \lambda+\beta \mu=0$, and that not all components of $\mathbf{u}_{1}$ will be determined.

- More generally, we then broaden the discussion to the case of semi-linear equations with A, B still constant, from the point of view of the existence of solutions and appropriateness of boundary data.

We start by considering $2 \times 2$ systems in Section 2 , and then look at $3 \times 3$ systems in Sections 3 - 4. Although our discussion will make use of trivial linear algebra and partial differentiation, the combination of these ideas turns out to reveal some surprising results. 


\section{Two equations with two dependent variables}

With two dependent variables, with $\mathbf{f}=(f, g)^{\top}$, the system of PDEs is now just

$$
\begin{aligned}
& a_{11} \frac{\partial u}{\partial x}+a_{12} \frac{\partial v}{\partial x}+b_{11} \frac{\partial u}{\partial y}+b_{12} \frac{\partial v}{\partial y}=f \quad\left(=c_{11} u+c_{12} v \quad \text { in the linear homogeneous case }\right) \\
& a_{21} \frac{\partial u}{\partial x}+a_{22} \frac{\partial v}{\partial x}+b_{21} \frac{\partial u}{\partial y}+b_{22} \frac{\partial v}{\partial y}=g \quad\left(=c_{21} u+c_{22} v\right)
\end{aligned}
$$

with the coefficients $a_{i j}, b_{i j}, c_{i j}$ all constant. For the system not to be completely trivial (that is, for it not simply to be the pair of algebraic equations $(f=0, g=0)$, at least one of the coefficients on the left-hand side must be non-zero; we can thus take $a_{11}=1$ without loss of generality.

The degeneracy condition (1.6) holding for all $\lambda$ and $\mu$ here becomes

$$
|\mu \mathbf{A}-\lambda \mathbf{B}|=\mu^{2}|\mathbf{A}|+\lambda \mu\left(-a_{11} b_{22}+a_{21} b_{12}+a_{12} b_{21}-a_{22} b_{11}\right)+\lambda^{2}|\mathbf{B}|=0 .
$$

Hence both A and $\mathbf{B}$ are singular,

$$
|\mathbf{A}|=0, \quad|\mathbf{B}|=0,
$$

and, additionally,

$$
a_{11} b_{22}+a_{22} b_{11}=a_{12} b_{21}+a_{21} b_{12} .
$$

Note that with $a_{11}=1 \neq 0$, we are free to change our dependent variables, if necessary, to make $a_{12}=0$ and add a multiple of the first equation to the second (if necessary) to make $a_{21}=0$. The first part of (2.2) reduces to $a_{22}=0$ while (2.3) leads to $b_{22}=0$.

The coefficient matrices are now

$$
\mathbf{A}=\left(\begin{array}{ll}
1 & 0 \\
0 & 0
\end{array}\right), \quad \mathbf{B}=\left(\begin{array}{cc}
b_{11} & b_{12} \\
b_{21} & 0
\end{array}\right)
$$

A change of independent variables, replacing $(x, y)$ by $(\hat{x}, \hat{y})$ such that $x=\hat{x}, y=\hat{y}+b_{11} \hat{x}$, i.e. $\hat{x}=x, \hat{y}=y-b_{11} x$, gives

$$
\frac{\partial u}{\partial \hat{x}}=\frac{\partial u}{\partial x}+b_{11} \frac{\partial u}{\partial y}, \quad \frac{\partial u}{\partial \hat{y}}=\frac{\partial u}{\partial y}, \quad\left(\frac{\partial u}{\partial x}=\frac{\partial u}{\partial \hat{x}}-b_{11} \frac{\partial u}{\partial \hat{y}}, \quad \frac{\partial u}{\partial y}=\frac{\partial u}{\partial \hat{y}}\right),
$$

and this can be employed to ensure that $b_{11}$ vanishes, in which case the second of (2.2) gives $b_{12} b_{21}=0$.

Scaling (if needed) gives three canonical problems, for each of which $\operatorname{rank}(\mu \mathbf{A}-\lambda \mathbf{B})=$ 1. We discuss each in turn, first as a homogeneous constant-coefficient linear system, then as a semi-linear system, discussing all possible degeneracies. 
Type 2.1. $b_{12}=b_{21}=0$.

$$
\frac{\partial u}{\partial x}=f, \quad 0=g, \quad \mu \mathbf{A}-\lambda \mathbf{B}=\left(\begin{array}{cc}
\mu & 0 \\
0 & 0
\end{array}\right)
$$

Linear: Substituting the expansion (1.13) and equating coefficients of the most singular term, namely $\mathcal{H}_{1}^{\prime}(\lambda y-\mu x)$ (which is, of course, equal to $\mathcal{H}(\lambda y-\mu x)$ ), shows that, in the generic case of $c_{22} \neq 0, \mathbf{u}_{0}=\left(u_{0}, v_{0}\right)^{\top}$ can only be non-zero when $\mu=0$ (and hence $\alpha=0$ ). Thus there is a single family of 'characteristics' $y=$ constant. When we consider the next term in the expansion (1.13), we find that $\mathbf{u}_{0}$ vanishes unless

$$
|\mathbf{C}+\beta \mathbf{A}|=\left|\begin{array}{cc}
c_{11}+\beta & c_{12} \\
c_{21} & c_{22}
\end{array}\right|=0,
$$

so that $\mathbf{C}$ determines both the $x$-dependence of $\mathbf{u}$, via $\beta$, and its direction, i.e. the eigenvector $\left(u_{0}, v_{0}\right)$.

However, for $c_{22}=0$, various special cases can occur. In particular, if $c_{12}=c_{22}=0, v$ can take any values, with singularities along any curves; with $c_{21}=0=c_{12}=c_{22}, u$ again satisfies a first-order PDE with a $\mu=0$ characteristic, while for $c_{21} \neq 0=c_{12}=c_{22}, u \equiv 0$. Next, for $c_{22}=0, c_{21} \neq 0, c_{12} \neq 0, u \equiv v \equiv 0$ and, finally, with $c_{22}=c_{21}=0, c_{12} \neq 0$, both $u$ and $v$ are indeterminate although they are related through the first PDE.

Semi-linear: If $g$ depends on $v$, the second of (2.5) is solved to get

$$
v=V(x, y, u)
$$

and this is then substituted into the first of (2.5): $\partial u / \partial x=f$, which can be solved as a family of ODEs given initial data on a curve $y=Y(x) ; v$ is then determined from (2.6). It is clear that for us to have a solution, any initial data prescribed for $v$ must satisfy (2.6).

If $g$ is independent of $v$ but varies with $u$, the second of (2.5) is solved to get $u=U(x, y)$. Any data prescribed must be consistent with this. Then the first of (2.5) fixes $v$, provided that $f$ depends on $v$; specified data must be consistent. For $f$ independent of $v$, the first equation is either an identity or cannot hold.

If $g$ depends upon neither $u$ nor $v$, the second equation is either impossible to satisfy or an identity. In the latter case, at least $v$ is then indeterminate.

Type 2.2. $b_{12}=1, b_{21}=0$.

$$
\frac{\partial u}{\partial x}+\frac{\partial v}{\partial y}=f, \quad 0=g, \quad \mu \mathbf{A}-\lambda \mathbf{B}=\left(\begin{array}{cc}
\mu & -\lambda \\
0 & 0
\end{array}\right) .
$$

Linear: We now need $\mu c_{22}+\lambda c_{21}=0$ so that the single family of characteristics is now C-dependent, with a single eigenvector satisfying $c_{21} u_{0}+c_{22} v_{0}=0$, as long as $c_{21}$ and 
$c_{22}$ do not both vanish. In this case, $\mathbf{C}$ determines both the direction of propagation of singularities and the mode of propagation, i.e. the eigenvector $\mathbf{u}_{0}$. In the extra-degenerate case of $c_{21}=c_{22}=0, u$ and $v$ are indeterminate, and we can have any sort of singularities on any line, as long as they are compatible with $\partial u / \partial x+\partial v / \partial y=c_{11} u+c_{12} v$.

Semi-linear: With $g$ depending on $v$, the second equation can be solved and the result used in the first, to get a single, generally quasi-linear, PDE for $u$. Alternatively, $u$ dependence of $g$ can be used to eliminate $u$ and get a single PDE for $v$. In either case, any initial data must be consistent with $g=0$ for there to be a solution. Should $g$ not vary with either of $u$ or $v$, the second of (2.7) either (i) holds trivially, with the first not giving a unique solution for $u, v$, or (ii) fails to be satisfied so the problem has no solution.

Type 2.3. $b_{12}=0, b_{21}=1$.

$$
\frac{\partial u}{\partial x}=f, \quad \frac{\partial u}{\partial y}=g, \quad \mu \mathbf{A}-\lambda \mathbf{B}=\left(\begin{array}{cc}
\mu & 0 \\
-\lambda & 0
\end{array}\right) .
$$

Linear: We now have to consider the first two terms in (1.13). Taking $\mathbf{u}_{1}=\left(u_{1}, v_{1}\right)^{\top}$, this reveals that $u_{0}=0$ and $-\mu u_{1}=c_{12} v_{0}, \lambda u_{1}=c_{22} v_{0}$. Now it is only when $c_{12}$ and $c_{22}$ do not both vanish that there is a single family of $\mathbf{C}$-dependent characteristics $c_{22} y+c_{12} x=$ constant, with a single eigenvector $\left(0, v_{0}\right)^{\top}$. In this case, $\mathbf{C}$ again determines the direction and mode of singularity propagation. Again, with $c_{12}=c_{22}=0$, singularities in $v$ can be arbitrary.

Semi-linear: If neither $f$ nor $g$ has dependence on $v,(2.8)$ requires a compatibility condition,

$$
\frac{\mathrm{d}}{\mathrm{dy}} f=\frac{\partial f}{\partial y}+g \frac{\partial f}{\partial u}=\frac{\mathrm{d}}{\mathrm{dx}} g=\frac{\partial g}{\partial x}+f \frac{\partial g}{\partial u}
$$

to get $u$; $v$ is then indeterminate. If one, but not both, of $f$ and $g$ depends on $v$, the corresponding equation can be used to find $v$ in terms of $u$, with the other equation being an ODE for $u$; initial data for $v$ must be consistent. Should both $f$ and $g$ vary with $v$, $v$ can (in principle) be eliminated to get a (generally fully non-linear) PDE for $u$. This determines $u$ and then $v$ can be got from either part of (2.8); specified data has to be consistent.

It is clear that, in the forms written, Type 2.1 and Type 2.2 are algebraic-differential systems, while only differential equations make up Type 2.3 .

It is easily checked that introducing extra independent variables does not change these types of degenerate system. 


\section{Three equations with three dependent variables}

We now turn to non-trivial problems for $\mathbf{u}=(u, v, w)^{\top}$, with $\mathbf{A}$ and $\mathbf{B}$ both constant $3 \times 3$ matrices. The right-hand side is now $(f, g, h)^{\top}$, and in the homogeneous linear case

$$
\left(\begin{array}{l}
f \\
g \\
h
\end{array}\right)=\mathbf{C u}=\left(\begin{array}{l}
c_{11} u+c_{12} v+c_{13} w \\
c_{21} u+c_{22} v+c_{23} w \\
c_{31} u+c_{32} v+c_{33} w
\end{array}\right)
$$

We can again take $a_{11}=1$, then redefine the dependent variables and take linear combinations of the equations, if necessary, to make $a_{12}=a_{13}=a_{21}=a_{31}=0$.

There are now two main cases, since we still need $|\mathbf{A}|=|\mathbf{B}|=0$ so both matrices have rank less than three:

Case 1. $\operatorname{Rank}(\mathbf{A})=1$, so

$$
\mathbf{A}=\left(\begin{array}{lll}
1 & 0 & 0 \\
0 & 0 & 0 \\
0 & 0 & 0
\end{array}\right)
$$

Case 2. $\operatorname{Rank}(\mathbf{A})=2$, so after another change of dependent variables and another new combination of equations if needed,

$$
\mathbf{A}=\left(\begin{array}{lll}
1 & 0 & 0 \\
0 & 1 & 0 \\
0 & 0 & 0
\end{array}\right)
$$

We sub-divide Case 1 into sub-cases 1(i) and 1(ii) according to:

Case 1(i). $b_{22}=b_{23}=b_{32}=b_{33}=0$;

Case 1(ii). At least one of $b_{22}, b_{23}, b_{32}, b_{33}$ is non-zero. From $0=|\mu \mathbf{A}-\lambda \mathbf{B}|=\lambda^{2} \mu\left(b_{22} b_{33}-\right.$ $\left.b_{23} b_{32}\right)-\lambda^{3}|\mathbf{B}|$, we see that $b_{22} b_{33}=b_{23} b_{32}$, and further manipulation of the second and third rows and columns can be used to get $b_{22}=1, b_{23}=b_{32}=b_{33}=0$.

For Case 2, the coefficient of $\lambda \mu^{2}$ in the expansion of $|\mu \mathbf{A}-\lambda \mathbf{B}|$ is $-b_{33}$ so (1.6) gives $b_{33}=0$ and then

$$
|\mu \mathbf{A}-\lambda \mathbf{B}|=\left|\begin{array}{ccc}
\mu-\lambda b_{11} & -\lambda b_{12} & -\lambda b_{13} \\
-\lambda b_{21} & \mu-\lambda b_{22} & -\lambda b_{23} \\
-\lambda b_{31} & -\lambda b_{32} & 0
\end{array}\right|=-\lambda^{3}|\mathbf{B}|-\lambda^{2} \mu\left(b_{13} b_{31}+b_{23} b_{32}\right)=0
$$

with corresponding PDEs

$$
\begin{aligned}
& \frac{\partial u}{\partial x}+b_{11} \frac{\partial u}{\partial y}+b_{12} \frac{\partial v}{\partial y}+b_{13} \frac{\partial w}{\partial y}=f, \\
& v x+b_{21} \frac{\partial u}{\partial y}+b_{22} \frac{\partial v}{\partial y}+b_{23} \frac{\partial w}{\partial y}=g, \quad b_{31} \frac{\partial u}{\partial y}+b_{32} \frac{\partial v}{\partial y}=h .
\end{aligned}
$$


If $b_{13} \neq 0, b_{23} \neq 0$, we can take a linear combination of the first and second of (3.2) to get rid of the $\partial w / \partial y$ in the first equation. If $b_{13} \neq 0=b_{23}$, we can swap the first two equations, and $u$ with $v$. Hence we can always assume that $b_{13}=0$, while with a change of independent variables we may take $b_{11}=0$ :

$$
|\mathbf{B}|=\left|\begin{array}{ccc}
0 & b_{12} & 0 \\
b_{21} & b_{22} & b_{23} \\
b_{31} & b_{32} & 0
\end{array}\right|=b_{12} b_{23} b_{31}=0 \quad \text { and } \quad b_{23} b_{32}=0
$$

from (3.1). We can consider the following sub-cases:

Case 2(i). $b_{31}=0, b_{32} \neq 0$;

Case 2(ii). $b_{31}=0, b_{32}=0$;

Case 2(iii). $b_{31} \neq 0, b_{32} \neq 0$;

Case 2(iv). $b_{31} \neq 0, b_{32}=0$.

In the remainder of this section, we catalogue the canonical types, based on consideration of the left-hand sides of the equations. For each type we will only consider the basic degeneracies that can occur for the linear problem in which the right-hand side is Cu. Hence each type will not be covered in detail as in Section 2, where we discussed all the higher-order degeneracies. Discussion of the resulting semi-linear problems is left until Section 4.

We remark that the method of weights, as described in the Introduction, would immediately tell us that Type 3.1 - Type 3.3 below are in general equivalent to first-order scalar equations, while Type 3.4 - Type 3.13 are equivalent to second-order scalar equations. This already gives general clues about the types of singularity the systems can support and the boundary conditions they can satisfy. However, we shall find that there are many special cases to consider.

\subsection{Case 1(i)}

Combining the last two equations and the last two dependent variables as necessary, the system can be written as

$$
\frac{\partial u}{\partial x}+b_{11} \frac{\partial u}{\partial y}+b_{12} \frac{\partial v}{\partial y}=f, \quad b_{21} \frac{\partial u}{\partial y}=g, \quad 0=h,
$$

where $f, g$ and $h$ are (potentially) functions of both the independent variables $x$ and $y$ and the unknowns $u, v$ and $w$. Again making a change of variable $x=\hat{x}, y=\hat{y}+b_{11} \hat{x}$ if needed, the system may be supposed to be of the simpler form

$$
\frac{\partial u}{\partial x}+b_{12} \frac{\partial v}{\partial y}=f, \quad b_{21} \frac{\partial u}{\partial y}=g, \quad 0=h .
$$


Depending on whether or not $b_{12}$ or $b_{21}$ vanishes, there are then four possibilities, after further rescaling if needed:

Type 3.1. $b_{12}=b_{21}=0$.

$$
\frac{\partial u}{\partial x}=f, \quad 0=g, \quad 0=h, \quad \mu \mathbf{A}-\lambda \mathbf{B}=\left(\begin{array}{ccc}
\mu & 0 & 0 \\
0 & 0 & 0 \\
0 & 0 & 0
\end{array}\right) .
$$

With $\mathbf{u}_{0}=\left(u_{0}, v_{0}, w_{0}\right)^{\top}$ in (1.13), we have $\mu u_{0}=0$ and there appear to be two possibilities. However, for $u_{0}=0$, we require

$$
\left(\begin{array}{ll}
c_{22} & c_{23} \\
c_{32} & c_{33}
\end{array}\right)
$$

to be singular; we do not consider this special degeneracy further.

We are left with $\mu=0$, as in Type 2.1, but now needing $c_{22} c_{33} \neq c_{23} c_{32}$. Again, $\alpha=0$ and $\beta$ is determined from $\mathbf{C}$, now by the condition that

$$
|\mathbf{C}+\beta \mathbf{A}|=\left|\begin{array}{ccc}
c_{11}+\beta & c_{12} & c_{13} \\
c_{21} & c_{22} & c_{23} \\
c_{31} & c_{32} & c_{33}
\end{array}\right|=0
$$

which also determines the mode of propagation $\left(u_{0}, v_{0}, w_{0}\right)$ along the characteristics $y=$ constant.

Type 3.2. $b_{12}=1, b_{21}=0$.

$$
\frac{\partial u}{\partial x}+\frac{\partial v}{\partial y}=f, \quad 0=g, \quad 0=h, \quad \mu \mathbf{A}-\lambda \mathbf{B}=\left(\begin{array}{ccc}
\mu & -\lambda & 0 \\
0 & 0 & 0 \\
0 & 0 & 0
\end{array}\right) .
$$

For the generic case, in which

$$
\left|\begin{array}{ll}
c_{21} & c_{23} \\
c_{31} & c_{33}
\end{array}\right| \text { and }\left|\begin{array}{ll}
c_{22} & c_{23} \\
c_{32} & c_{33}
\end{array}\right|
$$

are not both zero, we have $-\mu u_{0}+\lambda v_{0}=0$, so that the characteristics are determined in terms of $\mathbf{C}$ by

$$
\left|\begin{array}{ccc}
-\mu & \lambda & 0 \\
c_{21} & c_{22} & c_{23} \\
c_{31} & c_{32} & c_{33}
\end{array}\right|=0
$$


with $\mathbf{u}_{0}$ being an eigenvector of this matrix.

Type 3.3. $b_{12}=0, b_{21}=1$.

$$
\frac{\partial u}{\partial x}=f, \quad \frac{\partial u}{\partial y}=g, \quad 0=h, \quad \mu \mathbf{A}-\lambda \mathbf{B}=\left(\begin{array}{ccc}
\mu & 0 & 0 \\
-\lambda & 0 & 0 \\
0 & 0 & 0
\end{array}\right) .
$$

For the generic case, in which

$$
\left|\begin{array}{ll}
c_{12} & c_{13} \\
c_{32} & c_{33}
\end{array}\right| \text { and }\left|\begin{array}{ll}
c_{22} & c_{23} \\
c_{32} & c_{33}
\end{array}\right|
$$

are not both zero, we have $u_{0}=0$ as in Type 2.3, and, since

$$
\begin{aligned}
& -\mu u_{1}=c_{12} v_{0}+c_{13} w_{0}, \\
& \lambda u_{1}=c_{22} v_{0}+c_{23} w_{0}, \\
& 0=c_{32} v_{0}+c_{33} w_{0}
\end{aligned}
$$

singularities can propagate in the $\mathbf{C}$-dependent direction given by

$$
\left|\begin{array}{ccc}
-\mu & c_{12} & c_{13} \\
\lambda & c_{22} & c_{23} \\
0 & c_{32} & c_{33}
\end{array}\right|=0
$$

with associated eigenvector $\left(0, v_{0}, w_{0}\right)^{\top}$.

Type 3.4. $b_{12}=b_{21}=1$.

$$
\frac{\partial u}{\partial x}+\frac{\partial v}{\partial y}=f, \frac{\partial u}{\partial y}=g, 0=h, \mu \mathbf{A}-\lambda \mathbf{B}=\left(\begin{array}{ccc}
\mu & -\lambda & 0 \\
-\lambda & 0 & 0 \\
0 & 0 & 0
\end{array}\right)
$$

We note that for the generic case of $c_{33} \neq 0, w$ can be eliminated to obtain a nondegenerate, but special, $2 \times 2$ system with a unique characteristic direction: $\lambda=0$ is a double root of the characteristic equation and the reduced problem for $u, v$ is of parabolic type.

For each of the above four types of system, whether linear or semi-linear, the solutions depend sensitively upon the right-hand sides of the equations, as in Type 2.1 - Type 2.3. 


\subsection{Case 1(ii)}

Here (1.6) gives

$$
0=|\mu \mathbf{A}-\lambda \mathbf{B}|=\left|\begin{array}{ccc}
\mu-\lambda b_{11} & -\lambda b_{12} & -\lambda b_{13} \\
-\lambda b_{21} & -\lambda & 0 \\
-\lambda b_{31} & 0 & 0
\end{array}\right|=\lambda^{3} b_{13} b_{31}
$$

so at least one of $b_{13}$ and $b_{31}$ vanishes. There are then three possibilities which can be written as follows:

$$
\text { For } b_{13}=b_{31}=0 \text {, }
$$

$$
\frac{\partial u}{\partial x}+b_{11} \frac{\partial u}{\partial y}+b_{12} \frac{\partial v}{\partial y}=f, \quad b_{21} \frac{\partial u}{\partial y}+\frac{\partial v}{\partial y}=g, \quad 0=h .
$$

Taking $\hat{v}=v+b_{21} u$ if $b_{21} \neq 0$ and with another change of independent variables if need be, the system becomes

$$
\frac{\partial u}{\partial x}+b_{12} \frac{\partial v}{\partial y}=f, \quad \frac{\partial v}{\partial y}=g, \quad 0=h .
$$

Then adding a multiple of the second equation to the first gives

Type 3.5. $b_{13}=0, b_{31}=0$.

$$
\frac{\partial u}{\partial x}=f, \quad \frac{\partial v}{\partial y}=g, \quad 0=h, \quad \mu \mathbf{A}-\lambda \mathbf{B}=\left(\begin{array}{ccc}
\mu & 0 & 0 \\
0 & -\lambda & 0 \\
0 & 0 & 0
\end{array}\right) .
$$

For the generic case in which $c_{33} \neq 0, u_{0}$ and $v_{0}$ cannot both vanish. The problem then has two characteristic directions, with $(\lambda, \mu)$ parallel to $(0,1)$ or $(1,0)$. We have the two possibilities:

(a) $\lambda=0, u_{0}=0$, which requires that

$$
\left|\begin{array}{cc}
c_{22}-\alpha & c_{23} \\
c_{32} & c_{33}
\end{array}\right|=0
$$

and $\left(0, v_{0}, w_{0}\right)^{\top}$ is a corresponding eigenvector. As in Type 3.1(b), C determines the mode of propagation and its $x$-dependence along the characteristics.

(b) $\mu=0, v_{0}=0$, which requires that

$$
\left|\begin{array}{cc}
c_{11}+\beta & c_{13} \\
c_{31} & c_{33}
\end{array}\right|=0
$$

and $\left(u_{0}, 0, w_{0}\right)^{\top}$ is a corresponding eigenvector; this situation is analogous to (a) above. 
For $b_{13}=0, b_{31}=1$,

$$
\frac{\partial u}{\partial x}+b_{11} \frac{\partial u}{\partial y}+b_{12} \frac{\partial v}{\partial y}=f, \quad b_{21} \frac{\partial u}{\partial y}+\frac{\partial v}{\partial y}=g, \quad \frac{\partial u}{\partial y}=h,
$$

which, proceeding much as for Type 3.5 , can be written as

Type 3.6. $b_{13}=0, b_{31}=1$.

$$
\frac{\partial u}{\partial x}=f, \quad \frac{\partial v}{\partial y}=g, \quad \frac{\partial u}{\partial y}=h, \quad \mu \mathbf{A}-\lambda \mathbf{B}=\left(\begin{array}{ccc}
\mu & 0 & 0 \\
0 & -\lambda & 0 \\
-\lambda & 0 & 0
\end{array}\right) .
$$

We have $\mu u_{0}=-\lambda v_{0}=-\lambda u_{0}=0$, with the following cases for the generic situation, $c_{33} \neq 0$ :

(a) $\lambda=0, u_{0}=0$, which leads to the same situation as Type 3.5(a) above.

(b) $u_{0}=v_{0}=0$ and

$$
-\mu u_{1}=c_{13} w_{0}, \quad \lambda v_{1}=c_{23} w_{0}, \quad \lambda u_{1}=c_{33} w_{0}
$$

so that $(\lambda, \mu)=\left(c_{33},-c_{13}\right)$ and singularities can occur across $c_{13} x+c_{33} y=$ constant with eigenvector $\left(0,0, w_{0}\right)^{\top}$.

For $b_{13}=1, b_{31}=0$,

$$
\frac{\partial u}{\partial x}+b_{11} \frac{\partial u}{\partial y}+b_{12} \frac{\partial v}{\partial y}+\frac{\partial w}{\partial y}=f, \quad b_{21} \frac{\partial u}{\partial y}+\frac{\partial v}{\partial y}=g, \quad 0=h,
$$

which, on replacing $v$ by $\hat{v}=v+b_{21} u$ and $w$ by $\hat{w}=w+b_{11} u+b_{12} v$ is then

Type 3.7. $b_{13}=1, b_{31}=0$.

$$
\frac{\partial u}{\partial x}+\frac{\partial w}{\partial y}=f, \quad \frac{\partial v}{\partial y}=g, \quad 0=h, \quad \mu \mathbf{A}-\lambda \mathbf{B}=\left(\begin{array}{ccc}
\mu & 0 & -\lambda \\
0 & -\lambda & 0 \\
0 & 0 & 0
\end{array}\right)
$$

Similar to Type 3.5 and Type 3.6, for the generic case in which $c_{33} \neq 0$ there are the following possibilities:

(a) $\lambda=0, u_{0}=0$; we retrieve Type 3.5(a), in which singularities can occur across $x=$ constant.

(b) $v_{0}=0$; we now find that $-\mu u_{0}+\lambda w_{0}=0$ and $c_{31} u_{0}+c_{33} w_{0}=0$, so that $\mathbf{C}$ defines both the eigenvectors and the characteristic directions. 


\subsection{Case 2(i)}

Now $b_{31}=0 \neq b_{32}$ so, because of $(3.1), b_{23}=0$, and the third equation may be scaled:

$$
\frac{\partial u}{\partial x}=f, \quad \frac{\partial v}{\partial x}+b_{21} \frac{\partial u}{\partial y}=g, \quad \frac{\partial v}{\partial y}=h .
$$

If $b_{21}=0$, this is equivalent to Type 3.6 , and otherwise the system can be rewritten as

Type 3.8. $b_{31}=0 \neq b_{32}, b_{23}=0$.

$$
\frac{\partial u}{\partial x}=f, \quad \frac{\partial v}{\partial x}+\frac{\partial u}{\partial y}=g, \quad \frac{\partial v}{\partial y}=h, \quad \mu \mathbf{A}-\lambda \mathbf{B}=\left(\begin{array}{ccc}
\mu & 0 & 0 \\
-\lambda & \mu & 0 \\
0 & -\lambda & 0
\end{array}\right)
$$

To lowest order, $-\mu u_{0}=\lambda u_{0}-\mu v_{0}=\lambda v_{0}=0$. Hence $u_{0}=v_{0}=0$ and, since

$$
\begin{aligned}
& -\mu u_{1}=c_{13} w_{0}, \\
& \lambda u_{1}-\mu v_{1}=c_{23} w_{0}, \\
& \lambda v_{1}=c_{33} w_{0},
\end{aligned}
$$

$\lambda$ and $\mu$ must satisfy

$$
\left|\begin{array}{ccc}
\mu & 0 & c_{13} \\
-\lambda & \mu & c_{23} \\
0 & -\lambda & c_{33}
\end{array}\right|=0
$$

giving

$$
c_{33} \mu^{2}+c_{23} \lambda \mu+c_{13} \lambda^{2}=0 .
$$

For the generic case in which not all of $c_{13}, c_{23}$ and $c_{33}$ vanish, we have the possibility of two characteristics, which may be real or, for the first time, complex, according to the sign of $c_{23}^{2}-4 c_{13} c_{33}$. However, only one eigenvector, namely $\left(0,0, w_{0}\right)^{\top}$, can propagate singularities, so the situation is far from that of conventional hyperbolicity, where two Riemann invariants are associated with two real characteristics.

\subsection{Case 2(ii)}

With $b_{31}=0=b_{32}, w$ may be redefined to give us

$$
\frac{\partial u}{\partial x}+b_{12} \frac{\partial v}{\partial y}=f, \quad \frac{\partial v}{\partial x}+\frac{\partial w}{\partial y}=g, \quad 0=h
$$

First suppose that $b_{23} \neq 0$.

If $b_{12}=0$ we get a system like Type 3.7 , while for $b_{12} \neq 0$ some rescaling leads to 
Type 3.9. $b_{31}=0=b_{32}, b_{12} \neq 0$.

$$
\frac{\partial u}{\partial x}+\frac{\partial v}{\partial y}=f, \quad \frac{\partial v}{\partial x}+\frac{\partial w}{\partial y}=g, \quad 0=h, \quad \mu \mathbf{A}-\lambda \mathbf{B}=\left(\begin{array}{ccc}
\mu & -\lambda & 0 \\
0 & \mu & -\lambda \\
0 & 0 & 0
\end{array}\right) .
$$

Since, to lowest order, $\mu u_{0}-\lambda v_{0}=\mu v_{0}-\lambda w_{0}=0$, the eigenvector $\mathbf{u}_{0}$ is determined by the propagation direction. But since

$$
\left(\begin{array}{c}
\mu u_{1}-\lambda v_{1} \\
\mu v_{1}-\lambda w_{1} \\
0
\end{array}\right)=-\left(\begin{array}{ccc}
c_{11}+\beta & c_{12}-\alpha & c_{13} \\
c_{21} & c_{22}+\beta & c_{23}-\alpha \\
c_{31} & c_{32} & c_{33}
\end{array}\right)\left(\begin{array}{c}
u_{0} \\
v_{0} \\
w_{0}
\end{array}\right)
$$

we require that $c_{31} u_{0}+c_{32} v_{0}+c_{33} w_{0}=0$. Hence, for the generic case in which $c_{32} \neq 0$, the propagation direction satisfies the quadratic equation $c_{31} \lambda^{2}+c_{32} \lambda \mu+c_{33} \mu^{2}=0$. However, in contrast to Type 3.8, there will generally be a different eigenvector for each propagation direction.

Returning to the general discussion, now take $b_{23}=0$ so the system is

$$
\frac{\partial u}{\partial x}+b_{12} \frac{\partial v}{\partial y}=f, \quad \frac{\partial v}{\partial x}+b_{21} \frac{\partial u}{\partial y}+b_{22} \frac{\partial v}{\partial y}=g, \quad 0=h .
$$

With $b_{12} \neq 0, b_{21} \neq 0$, appropriate scaling of $v$ and $y$ provides

Type 3.10. $b_{12} \neq 0, b_{21} \neq 0, b_{22} \neq 0$.

$$
\frac{\partial u}{\partial x} \pm \frac{\partial v}{\partial y}=f, \quad \frac{\partial v}{\partial x}+\frac{\partial u}{\partial y}+b_{22} \frac{\partial v}{\partial y}=g, \quad 0=h, \quad \mu \mathbf{A}-\lambda \mathbf{B}=\left(\begin{array}{ccc}
\mu & \mp \lambda & 0 \\
-\lambda & \mu-\lambda b_{22} & 0 \\
0 & 0 & 0
\end{array}\right)
$$

The sign for the second term in the first equation is that of $b_{12} b_{21}$. It is not possible to rescale again to remove the constant $b_{22}$.

Now, through $b_{22}$, B plays an additional role because

$$
-\mu u_{0} \pm \lambda v_{0}=0, \quad \lambda u_{0}+\left(\lambda b_{22}-\mu\right) v_{0}=0,
$$

so that $\mu^{2}-\left(b_{22} \mu \pm \lambda\right) \lambda=0$. Note that, through $w_{0}, \mathbf{C}$ still plays a role in determining the eigenvectors, and can also be responsible for higher degeneracy of non-generic cases.

Returning again to the general discussion, with $b_{12}=0 \neq b_{21}$, scaling gives

$$
\frac{\partial u}{\partial x}=f, \quad \frac{\partial v}{\partial x}+\frac{\partial u}{\partial y}+b_{22} \frac{\partial v}{\partial y}=g, \quad 0=h .
$$


If $b_{22}=0$ this is equivalent to Type 3.4. However, for $b_{22} \neq 0$, scaling to make $b_{22}=1$, adding the first equation to the second, replacing $v$ by $\hat{v}=u+v$, and changing the independent variables leads to

$$
\frac{\partial u}{\partial x}=f, \quad \frac{\partial v}{\partial y}=g, \quad 0=h,
$$

which is the same as Type 3.5.

Next, for $b_{12} \neq 0=b_{21}$, the system is, after some scaling,

$$
\frac{\partial u}{\partial x}+\frac{\partial v}{\partial y}=f, \quad \frac{\partial v}{\partial x}+b_{22} \frac{\partial v}{\partial y}=g, \quad 0=h .
$$

With $b_{22}=0$ this is equivalent to Type 3.4. For $b_{22} \neq 0$, we have, with a possible change of y,

$$
\frac{\partial u}{\partial x}+\frac{\partial v}{\partial y}=f, \quad \frac{\partial v}{\partial x}+\frac{\partial v}{\partial y}=g, \quad 0=h .
$$

Subtracting the second equation from the first, replacing $u$ by $\hat{u}=u-v$, and a change of independent variables leads to

$$
\frac{\partial u}{\partial x}=f, \quad \frac{\partial v}{\partial y}=g, \quad 0=h,
$$

which is again the same as Type 3.5.

Finally for this sub-case, $b_{12}=0=b_{21}$ and

$$
\frac{\partial u}{\partial x}=f, \quad \frac{\partial v}{\partial x}+b_{22} \frac{\partial v}{\partial y}=g, \quad 0=h .
$$

For $b_{22} \neq 0$, a change of independent variable makes the system identical to Type 3.5 once again. However, if $b_{22}=0$, we have a new type:

Type 3.11. $B=0$.

$$
\frac{\partial u}{\partial x}=f, \quad \frac{\partial v}{\partial x}=g, \quad 0=h, \quad \mu \mathbf{A}-\lambda \mathbf{B}=\left(\begin{array}{ccc}
\mu & 0 & 0 \\
0 & \mu & 0 \\
0 & 0 & 0
\end{array}\right)
$$

Since $\mu u_{0}=\mu v_{0}=0$, we have $\mu=0$ and the characteristics are $y=$ constant. However, we now require that

$$
(\mathbf{C}+\beta \mathbf{A}) \mathbf{u}_{0}=\left(\begin{array}{ccc}
c_{11}+\beta & c_{12} & c_{13} \\
c_{21} & c_{22}+\beta & c_{23} \\
c_{31} & c_{32} & c_{33}
\end{array}\right) \mathbf{u}_{0}=\mathbf{0},
$$

so that, for the generic case of $c_{33} \neq 0, \mathbf{C}$ again determines the eigenvector and the exponential variation of the solution along the characteristic. 


\subsection{Case 2(iii)}

Now $b_{31} \neq 0, b_{32} \neq 0$ and so $b_{23}=0$ from (3.3):

$$
\frac{\partial u}{\partial x}+b_{12} \frac{\partial v}{\partial y}=f, \quad \frac{\partial v}{\partial x}+b_{21} \frac{\partial u}{\partial y}+b_{22} \frac{\partial v}{\partial y}=g, \quad \frac{\partial u}{\partial y}+\frac{\partial v}{\partial y}=h,
$$

with appropriate scaling. Subtracting a multiple of the third equation from the second yields

$$
\frac{\partial u}{\partial x}+b_{12} \frac{\partial v}{\partial y}=f, \quad \frac{\partial v}{\partial x}+b_{21} \frac{\partial u}{\partial y}=g, \quad \frac{\partial u}{\partial y}+\frac{\partial v}{\partial y}=h .
$$

Taking $b_{12}=b_{21}=0$, replacing $v$ by $\hat{v}=u+v$, and adding the first equation to the second gives a problem equivalent to Type 3.6.

If $b_{12} \neq 0=b_{21}$, with suitable scaling, the system is

$$
\frac{\partial u}{\partial x}+\frac{\partial v}{\partial y}=f, \quad \frac{\partial v}{\partial x}=g, \quad \frac{\partial u}{\partial y}+\frac{\partial v}{\partial y}=h .
$$

Replacing $u$ by $\hat{u}=u+v$ and adding the second equation to the first shows that this system is equivalent to Type 3.8 .

For $b_{12}=0 \neq b_{21}$, with suitable scaling, the system is

$$
\frac{\partial u}{\partial x}=f, \quad \frac{\partial v}{\partial x}+\frac{\partial u}{\partial y}=g, \quad \frac{\partial u}{\partial y}+\frac{\partial v}{\partial y}=h
$$

Manipulations as immediately above indicate that this is also equivalent to Type 3.8.

With $b_{12} \neq 0, b_{21} \neq 0$, we can write the system as

$$
\frac{\partial u}{\partial x}+\frac{\partial v}{\partial y}=f, \quad \frac{\partial v}{\partial x}+b_{21} \frac{\partial u}{\partial y}=g, \quad \frac{\partial u}{\partial y}+\frac{\partial v}{\partial y}=h .
$$

A change of independent variables and the subtraction of the third equation from the first produce

$$
\frac{\partial u}{\partial x}-\left(1+b_{21}\right) \frac{\partial u}{\partial y}=f, \quad \frac{\partial v}{\partial x}=g, \quad \frac{\partial u}{\partial y}+\frac{\partial v}{\partial y}=h .
$$

Taking $b_{21}=-1$, replacing $v$ by $\hat{v}=u+v$, and adding the first equation to the second gives a system equivalent to (3.16). Trying $b_{21} \neq-1$, the problem scales to

$$
\frac{\partial u}{\partial x}+\frac{\partial u}{\partial y}=f, \quad \frac{\partial v}{\partial x}=g, \quad \frac{\partial u}{\partial y}+\frac{\partial v}{\partial y}=h .
$$

Replacing $u$ by $\hat{u}=u+v$, adding the second equation to the first and subtracting the third from the first leads to a problem like Type 3.8. 


\subsection{Case 2(iv)}

Here $b_{31} \neq 0=b_{32}$ and, because $b_{31} \neq 0$, (3.3) gives $b_{12}=0$ and /or $b_{23}=0$ in

$$
\frac{\partial u}{\partial x}+b_{12} \frac{\partial v}{\partial y}=f, \quad \frac{\partial v}{\partial x}+b_{22} \frac{\partial v}{\partial y}+b_{23} \frac{\partial w}{\partial y}=g, \quad \frac{\partial u}{\partial y}=h,
$$

where the third equation has been used to simplify the second.

For $b_{12}=b_{23}=0$, adding a multiple of the third equation to the second puts it in the form

$$
\frac{\partial u}{\partial x}=f, \quad \frac{\partial v}{\partial x}+b_{22} \frac{\partial v}{\partial y}=g, \quad \frac{\partial u}{\partial y}=h .
$$

For $b_{22}=0$ this is equivalent to Type 3.6, while for $b_{22} \neq 0$, the problem scales to

Type 3.12. $b_{22} \neq 0, b_{23}=0, b_{31} \neq 0$.

$$
\frac{\partial u}{\partial x}=f, \quad \frac{\partial v}{\partial x}+\frac{\partial v}{\partial y}=g, \quad \frac{\partial u}{\partial y}=h, \quad \mu \mathbf{A}-\lambda \mathbf{B}=\left(\begin{array}{ccc}
\mu & 0 & 0 \\
0 & \mu-\lambda & 0 \\
-\lambda & 0 & 0
\end{array}\right)
$$

To lowest order, $-\mu u_{0}=(-\mu+\lambda) v_{0}=\lambda u_{0}=0$ and thus we generically have $u_{0}=v_{0}=0$, which implies that

$$
-\mu u_{1}=c_{13} w_{0}, \quad(-\mu+\lambda) v_{1}=c_{23} w_{0}, \quad \lambda u_{1}=c_{33} w_{0} .
$$

Hence

$$
\left|\begin{array}{ccc}
-\mu & 0 & -c_{13} \\
0 & -\mu+\lambda & -c_{23} \\
\lambda & 0 & -c_{33}
\end{array}\right|=0
$$

giving $(\lambda-\mu)\left(c_{33} \mu+c_{13} \lambda\right)=0$. Clearly, for the generic case of $c_{13}, c_{33}$ not both zero, we have another case of two real characteristics; they are coincident if $c_{13}=-c_{33}$.

Returning for the last time to the general discussion, taking $b_{23}=0 \neq b_{12}$ leads to

$$
\frac{\partial u}{\partial x}+b_{12} \frac{\partial v}{\partial y}=f, \quad \frac{\partial v}{\partial x}+b_{22} \frac{\partial v}{\partial y}=g, \quad \frac{\partial u}{\partial y}=h .
$$

A change of independent variable, addition of a multiple of the third equation to the first and a scaling give a problem equivalent to Type 3.8.

For $b_{23} \neq 0=b_{12}$, a redefinition of $w$ puts the system into the form

Type 3.13. $b_{22}=0, b_{23} \neq 0, b_{31} \neq 0$.

$$
\frac{\partial u}{\partial x}=f, \quad \frac{\partial v}{\partial x}+\frac{\partial w}{\partial y}=g, \quad \frac{\partial u}{\partial y}=h, \quad \mu \mathbf{A}-\lambda \mathbf{B}=\left(\begin{array}{ccc}
\mu & 0 & 0 \\
0 & \mu & -\lambda \\
-\lambda & 0 & 0
\end{array}\right) .
$$


In this situation, $-\mu u_{0}=-\mu v_{0}+\lambda w_{0}=\lambda u_{0}=0$, giving $u_{0}=0, \lambda w_{0}=\mu v_{0}$. Generically this implies that

$-\mu u_{1}=c_{12} v_{0}+c_{13} w_{0}, \quad-\mu v_{1}+\lambda w_{1}-\beta u_{0}+\alpha w_{0}=c_{22} v_{0}+c_{23} w_{0}, \quad \lambda u_{1}=c_{32} v_{0}+c_{33} w_{0}$.

Hence singularities can propagate as long as $\left(c_{12} v_{0}+c_{13} w_{0}\right) \lambda=-\left(c_{32} v_{0}+c_{33} w_{0}\right) \mu$, and we see that there are two families of characteristics, determined in terms of $\mathbf{C}$ by $c_{33} \mu^{2}+$ $\left(c_{13}+c_{32}\right) \lambda \mu+c_{12} \lambda^{2}=0$; the respective eigenvectors depend correspondingly on $\mathbf{C}$. This generic case demands that at least one of $c_{33}, c_{13}+c_{32}$ and $c_{12}$ be non-zero, along with $c_{12} c_{33} \neq c_{13} c_{32}$, see Section 4.2 .

This system has such a wide variety of higher-order degeneracies that we will discuss it in further detail in Section 4.

We note that the system (1.8) is of this type.

\subsection{Summary}

Concerning singularity propagation, the most striking feature of the above catalogue are the roles played by

(i) the degeneracy of the matrix $\mu \mathbf{A}-\lambda \mathbf{B}$ in determining the propagation directions, and

(ii) the matrix $\mathbf{C}$ which usually decides the propagation directions and always decides the propagating eigenmodes.

Concerning the directions of propagation, we can classify the generic degeneracies according to :

1. Type 3.1 - Type 3.3 have a single characteristic, generalising the cases of Type 2.1 Type 2.3.

2. Type 3.4 and Type 3.11 have one double characteristic.

3. Type 3.5 - Type 3.7, Type 3.10 and Type 3.12 have two real characteristics.

4. Type 3.8, Type 3.9 and Type 3.13 have two, possibly complex, characteristics.

\section{Summary of three-by-three system types}

We now briefly generalise our discussion of the systems listed above to situations where they are semi-linear.

\subsection{Semi-linear degenerate systems}

We first note some obvious facts regarding Type 3.1 - Type 3.7.

- Type 3.1 is generically equivalent to Type 2.1, needing two constraints on the data. 
- Type 3.2 is generically equivalent to Type 2.2, needing two constraints on the data.

- Type 3.3 is generically equivalent to Type 2.3, needing two constraints on the data.

- Type 3.4 is generically equivalent to a $2 \times 2$ system, needing one constraint on the Cauchy data.

- Type 3.5 is generically equivalent to a $2 \times 2$ system, needing one constraint on the Cauchy data.

- Type 3.6 is rather like Type 2.3 for $2 \times 2$ systems; unless $w$ appears nowhere in the right-hand sides of (3.15); $w$ can be eliminated to get a generally fully non-linear $2 \times 2$ system, needing one constraint on the Cauchy data. If $w$ is completely absent from the system, a compatibility condition must be satisfied by the equations.

- Type 3.7, for the generic case of $h$ depending upon $w$, is again equivalent to a (quasilinear) $2 \times 2$ system, needing one constraint on the Cauchy data.

The different cases can be further categorised as follows:

Type 3.1 - Type 3.3 The first three canonical forms are differential-algebraic equations and doubly degenerate, turning out to be generically equivalent to $2 \times 2$ degenerate problems: Provided that $h$ depends upon $w, w$ can be found from the third equation and substituted into the first two to get a degenerate pair of PDEs for $u$ and $v$.

Should $h$ be independent of $w$, unless $h$ is identically constant, $u$ or $v$ can then be got from the third equation and eliminated from the first two, to get a pair of equations involving derivatives of the remaining unknown, $v$ or $u$ respectively. With $w$ appearing in neither $f$ nor $g$, this reduced problem is as for the corresponding $2 \times 2$ case. With $w$ in one but not the other, there is one semi-linear equation which can be solved for the surviving unknown, and the other two unknowns are got from the other two equations. With $w$ in both $f$ and $g$, it is eliminated to get a (generally quasi-linear) PDE for the survivor of $u$ and $v$; the other and $w$ are then determined explicitly.

For these problems, only one set of Cauchy data can be imposed.

Type 3.4, Type 3.5, Type 3.10 and Type 3.11 The last equation is again algebraic and no derivatives of $w$ appear. It is easily checked that the first two PDEs form a non-degenerate system, regarded as a problem for $u$ and $v$ alone. This means that as long as $h$ depends on $w, w$ can be eliminated and we have a $2 \times 2$ system. Two sets of Cauchy data are required.

If $h$ does not depend on $w$ then, excluding the trivial possibility of it being a function of $x$ and $y$ only, it must depend on one of the remaining dependent variables, say $u$. Using the last equation to eliminate $u$ from the first two equations gives two equations involving $v$ and its derivatives. For Type 3.10 it is possible that this pair of equations has extra degeneracy, with the two left-hand sides being proportional. If precisely one of $f$ and $g$ depends on $w$, we have one equation for $v$ alone; this gives $v$ and the other two equations then determine $u$ and $w$. If both $f$ and $g$ depend on $w, w$ is eliminated to give a single (possibly fully non-linear) PDE for $v$; the solution of this can again be used in the other equations to provide $u$ and $w$. In both these cases with $h$ independent of $w$, we should only impose one set of Cauchy data. 
If none of $f, g$ and $h$ depends upon $w$, for there to be a solution the equations must be consistent. For example, with the left-hand sides of the first two equations proportional, the same constant of proportionality must clearly relate the right-hand sides. The data for $u$ and $v$ must be consistent and $w$ is indeterminate.

Type 3.7 and Type 3.9 These are also differential-algebraic equations, with their third equation being algebraic, but, in contrast to those just considered, they contain a derivative of $w$ in their first two equations. For $h$ dependent on at least one of the dependent variables, say $w, w$ may be found from the last equation and substituted into the first two equations to get a (generally quasi-linear) $2 \times 2$ system. The other unknowns are found from this system and they then fix $w$. Clearly only two sets of Cauchy data are needed. If $h=0$ gives $w$ as a linear combination of $u$ and $v$, this elimination procedure could produce a degenerate $2 \times 2$ system, as in Section 2 .

Type 3.6, Type 3.8 and Type 3.12 These have derivatives in all three equations, and so are purely differential equations, but no derivative of $w$ appears. Should precisely one of $f, g$ and $h$ depend on $w$, the corresponding equation can fix $w$, with the other two PDEs forming a $2 \times 2$ system for $u$ and $v$. For Type 3.6 and Type 3.12, if it is $g$ which depends on $w$ so that the first and last equation make up the $2 \times 2$ system, this will then be degenerate, as in Section 2. Otherwise the $2 \times 2$ system is non-degenerate and two sets of Cauchy data are required.

With at least two of $f, g$ and $h$ depending upon $w, w$ is eliminated to give a (generally fully non-linear) problem for $u$ and $v$; two sets of conditions are imposed. Should the $2 \times 2$ system be linear or semi-linear, it is possible that this is degenerate, as in Section 2.

If none of $f, g$ and $h$ varies with $w$, some consistency condition is needed between the PDEs and $w$ is indeterminate.

Type 3.13 We are left with this as the only case with both:

- all three equations containing derivatives;

- derivatives of all dependent variables in the system.

It is clearly another purely partial differential-equation system. Its special status motivates the more detailed consideration in Section 4.2 below.

\subsection{Problems of Type 3.13}

The problem has the general form

$$
\begin{aligned}
& \frac{\partial u}{\partial x}=f \\
& \frac{\partial v}{\partial x}+\frac{\partial w}{\partial y}=g \\
& \frac{\partial u}{\partial y}=h .
\end{aligned}
$$


The generic case has $f$ and $h$ dependent on $v$ and $w$ so that (4.1) and (4.3) can be solved to get $v$ and $w$ in terms of $u$ and its derivatives. Substitution into (4.2) gives a second-order (usually quasi-linear) PDE for $u$. Depending on the nature of this equation, we would normally expect to impose either two initial conditions or one boundary condition.

A more special case is when (4.1) and (4.3) themselves add a further level of degeneracy, so that (4.1) and (4.3) provide a first-order PDE for $u$, and an equation relating $v$ and $w$ (and $u, \partial u / \partial x$ and/or $\partial u / \partial y$ ). This PDE is solved and substitution for $v$ (or $w$ ) in (4.2) gives another PDE for $w$ (or $v$ ). Two sets of Cauchy data are needed.

The most degenerate case has both $f$ and $h$ independent of $v$ and $w$. A compatibility condition for $f$ and $h$ has to hold. Assuming that it does, $u$ can be found (applying one initial condition) but $v$ and $w$ are indeterminate.

To see more clearly what might happen, we look at cases which are linear and, for simplicity, autonomous, so that

$$
\mathbf{f}=\mathbf{C u},
$$

with $\mathbf{C}$ constant. As before, $\mathbf{u}=(u, v, w)^{\top}$ and $\mathbf{f}=(f, g, h)^{\top}$.

The generic case has $c_{12} c_{33} \neq c_{13} c_{32}$ so that the subsidiary matrix

$$
\mathbf{C}_{s}=\left(\begin{array}{ll}
c_{12} & c_{13} \\
c_{32} & c_{33}
\end{array}\right)
$$

has rank 2, and (4.1) and (4.3) can be solved to get

$$
v=\left[\left(c_{33} \frac{\partial u}{\partial x}-c_{13} \frac{\partial u}{\partial y}\right)+\left(c_{13} c_{31}-c_{11} c_{33}\right) u\right] /\left(c_{12} c_{33}-c_{13} c_{32}\right)
$$

and

$$
w=\left[\left(c_{12} \frac{\partial u}{\partial y}-c_{32} \frac{\partial u}{\partial x}\right)+\left(c_{11} c_{32}-c_{12} c_{31}\right) u\right] /\left(c_{12} c_{33}-c_{13} c_{32}\right) .
$$

Substitution into (4.2) gives a PDE

$$
c_{33} \frac{\partial^{2} u}{\partial x^{2}}-\left(c_{32}+c_{13}\right) \frac{\partial^{2} u}{\partial x \partial y}+c_{12} \frac{\partial^{2} u}{\partial y^{2}}=d_{1} u+d_{2} \frac{\partial u}{\partial x}+d_{3} \frac{\partial u}{\partial y}
$$

where the $d_{i}$ are constant. This equation is clearly

- elliptic if $4 c_{12} c_{33}>\left(c_{13}+c_{32}\right)^{2}$,

- hyperbolic if $4 c_{12} c_{33}<\left(c_{13}+c_{32}\right)^{2}$,

- either parabolic or a second-order ODE if $4 c_{12} c_{33}=\left(c_{13}+c_{32}\right)^{2}$.

(Note that, for the generic case, at least one of $c_{12}, c_{13}, c_{32}$ and $c_{33}$ is non-zero.)

We remark that, had we used the method of weights described in the Introduction, we would have been immediately led to the differential operator on the left-hand side of (4.5), and hence identified that (1.12) is elliptic. 
The last case admits the the extra-degenerate possibility of $c_{12}=c_{33}=0$ with $c_{13}=$ $-c_{32} \neq 0$. Here $v$ and $w$ can be eliminated to get the first-order hyperbolic equation for $u$ :

$$
\left(c_{23}-c_{31}\right) \frac{\partial u}{\partial x}+\left(c_{11}-c_{22}\right) \frac{\partial u}{\partial y}=\left(c_{23} c_{11}-c_{22} c_{31}+c_{32} c_{21}\right) u
$$

The characteristic direction is $(\lambda, \mu)=\left(\left(c_{23}-c_{31}\right),\left(c_{11}-c_{22}\right)\right)$.

For $c_{12} c_{33}=c_{13} c_{32}$ with at least one of $c_{12}, c_{13}, c_{32}, c_{33}$ non-zero, say $c_{12}$, so that $\mathbf{C}_{s}$ now has rank 1 , we have

$$
c_{32} \frac{\partial u}{\partial x}-c_{12} \frac{\partial u}{\partial y}=\left(c_{11} c_{32}-c_{12} c_{31}\right) u
$$

with

$$
v=\left(\frac{\partial u}{\partial x}-c_{11} u-c_{13} w\right) / c_{12}
$$

The PDE (4.6) is solved for $u$, then (4.7) is substituted into (4.2) to give

$$
\frac{\partial w}{\partial y}-\frac{c_{13}}{c_{12}} \frac{\partial w}{\partial x}+\left(\frac{c_{13} c_{22}}{c_{12}}-c_{23}\right) w=\left(c_{21}-\frac{c_{22} c_{11}}{c_{12}}\right) u+\left(\frac{c_{22}}{c_{12}}+\frac{c_{11}}{c_{12}}\right) \frac{\partial u}{\partial x}-\frac{1}{c_{12}} \frac{\partial^{2} u}{\partial x^{2}} .
$$

This may be solved to find $w$ and hence $v$. (The appearance of the second derivative of $u$ in the PDE for $w$ might indicate that care should be taken in a numerical method based on this way of solving the system.)

The most special case is when $\mathbf{C}_{s}=\mathbf{0}$ so that

$$
\frac{\partial u}{\partial x}=c_{11} u, \quad \frac{\partial u}{\partial y}=c_{31} u .
$$

In this case $v$ and $w$ cannot be determined. (Note that if the problem were to be generalised by including constant terms on the right-hand sides of (4.1), (4.3), a solution might not exist. Supposing now that $\partial u / \partial x=c_{11} u+c_{10}$ and $\partial u / \partial y=c_{31} u+c_{30}$, the compatibility condition $c_{11} c_{30}=c_{31} c_{10}$ has to be satisfied to get a solution.)

We conclude this sub-section with a few specific examples.

4.2.1 $\frac{\partial u}{\partial x}=0, \frac{\partial u}{\partial y}=w, \frac{\partial v}{\partial x}+\frac{\partial w}{\partial y}=0$.

Solving directly gives $u=U(y), w=U^{\prime}(y), v=V(y)-U^{\prime \prime}(y) x$, with $U$ and $V$ being fixed by two boundary conditions.

In this case $c_{12} c_{33}=c_{13} c_{32}=0 \neq c_{33}$ so the system has one extra level of degeneracy.

4.2.2 $\frac{\partial u}{\partial x}=w, \frac{\partial u}{\partial y}=v, \frac{\partial v}{\partial x}+\frac{\partial w}{\partial y}=0$.

The system has general solution $v=V(y), w=W(x), u=\int{ }^{x} W(\xi) \mathrm{d} \xi+\int{ }^{y} V(\eta) \mathrm{d} \eta$ and is clearly hyperbolic. 
4.2.3 $\frac{\partial u}{\partial x}= \pm v, \frac{\partial u}{\partial y}=w, \frac{\partial v}{\partial x}+\frac{\partial w}{\partial y}=u$.

Here

$$
\frac{\partial^{2} u}{\partial y^{2}} \pm \frac{\partial^{2} u}{\partial x^{2}}=u
$$

which illustrates how easily degenerate systems can conceal ellipticity and hyperbolicity.

\section{Acknowledgments}

The authors would like to thank the referees for their comments, particularly for drawing their attention to the method of weights in [4].

\section{References}

[1] Ockendon J., Howison S., Lacey A., and Movchan A., Applied Partial Differential Equations, Oxford University Press, revised edition, 2003.

[2] Chester C. R., Techniques in Partial Differential Equations, McGraw-Hill, 1971.

[3] Agmon S., Douglis A., and Nirenberg L., Estimates near the boundary for solutions of elliptic partial differential equations satisfying general boundary conditions I, Comm. Pure Appl. Math., 12 (1959), 623-727.

[4] Agmon S., Douglis A., and Nirenberg L., Estimates near the boundary for solutions of elliptic partial differential equations satisfying general boundary conditions II, Comm. Pure Appl. Math., 17 (1964), 35-92.

[5] Martinson W. S. and Barton P. I., A differentiation index for partial differential-algebraic equations, SIAM Jl. Sci. Comp., 21 (6) (2000), 2295-2315. 